\title{
ARTÍCULOS
}

\section{INCIDENCIA DE LA EDAD DE ACCESO A LAS TECNOLOGÍAS DE LA INFORMACIÓN Y LA COMUNICACIÓN Y EL USO DE INTERNET EN EL APRENDIZAJE EN CIENCIAS*}

\author{
Denise Vaillant ${ }^{1}$ (D) \\ EDUARdo RodrígueZ-ZIDÁN ${ }^{1}$ (1) \\ Juan Pablo Zorrilla-Salgador ${ }^{1}$ (D)
}

\begin{abstract}
RESUMEN: El artículo presenta los resultados de una investigación referida a la incidencia de la edad de acceso a las Tecnologías de la Información y la Comunicación (TIC) y el uso temprano de Internet en los aprendizajes de ciencias por parte de jóvenes de 15 años de América Latina y el Caribe. Los datos se obtienen a partir de la base de datos de Programa Internacional para la Evaluación de Estudiantes (PISA) 2015 y mediante un método descriptivo de variables y la utilización de modelos de regresión logística. Los resultados evidencian que quienes usaron la computadora antes de los 6 años, tuvieron mejores resultados que aquellos que empezaron a mayor edad. Se constata también que existe un efecto positivo y significativo entre el uso intensivo de las TIC fuera de las instituciones educativas y el aprendizaje de competencias científicas.
\end{abstract}

Palabras clave: Competencias científicas. América Latina. Programa Internacional para la Evaluación de Estudiantes 2015. Uso intensivo de las tecnologías de la información y la comunicación.

\section{INCIDENCE OF THE AGE OF ACCESS TO INFORMATION AND COMMUNICATIONS TECHNOLOGY AND THE USE OF THE INTERNET IN SCIENCE LEARNING}

ABSTRACT: The paper presents the results of an investigation regarding the incidence of the age of access to the Information and Communications

\footnotetext{
*En este artículo se presentan algunos avances y resultados referidos al proyecto "Políticas docentes efectivas para un aprendizaje profundo" llevado a cabo por los autores en el Instituto de Educación de la Universidad ORT Uruguay bajo la dirección científica de la Dra. Denise Vaillant. El proyecto refiere al diseño de políticas docentes efectivas que promuevan un aprendizaje profundo con el apoyo de tecnologías digitales en ALyC. Dicho proyecto busca mejorar la comprensión actual acerca de las políticas docentes, las prácticas pedagógicas y los procesos de aprendizaje basados en tecnologías digitales en los sistemas educativos del continente. ${ }^{1}$ Universidad ORT Uruguay - Montevideo, Uruguay. E-mails: vaillant@ort.edu.uy; rodriguez_ce@ort.edu.uy; juanpablozorrilla@gmail.com DOI: 10.1590/ES0101-73302019199206
} 
Technology (ICT) and the early use of the Internet in the learning of sciences by 15-year-old teenagers from Latin America and Caribbean. The data was obtained from the Programme for International Student Assessment (PISA) 2015 database, by a descriptive method of variables and the use of logistic regression models. The results show that those who used the computer before the age of 6 had better results than those who started older. It is also noted that there is a positive and significant effect between the intensive use of ICT outside educational institutions and the learning of scientific competences.

Keywords: Scientific competences. Latin America. Programme for International Student Assessment 2015. Intensive use of information and communications technology.

\title{
INCIDÊNCIA DA IDADE DE ACESSO ÀS TECNOLOGIAS DA INFORMAÇÃo E COMUNICAÇÃo E A UTILIZAÇÃO DA INTERNET NA APRENDIZAGEM EM CIÊNCIAS
}

\begin{abstract}
RESUMO: O artigo apresenta os resultados de uma investigação acerca da incidência da idade de acesso às Tecnologias da Informação e Comunicação (TIC) e da utilização precoce da Internet nas aprendizagens de ciências por parte de jovens de 15 anos da América Latina e do Caribe. As informaçóes foram obtidas por meio da base de dados do Programa Internacional de Avaliação de Estudantes (PISA) 2015 e de um método descritivo de variáveis e de modelos de regressão logística. Os resultados evidenciaram que quem utilizou o computador antes dos 6 anos teve melhores resultados que aqueles que começaram a usá-lo com mais idade. Constatou-se também que existe um efeito positivo e significativo entre a utilização intensiva das TICs fora das instituiçóes educativas e a aprendizagem de competências científicas.
\end{abstract}

Palavras-chave: Competências científicas. América Latina. Programa Internacional para a Avaliação de Estudantes 2015. Uso intensivo das tecnologias da informação e comunicação.

\section{Introducción}

A

ctores políticos, investigadores, funcionarios de los ministerios de educación y de los organismos internacionales se interrogan acerca de los avances que registra hoy la economía digital y el uso de tecnologías en nuestras sociedades. Al respecto podríamos preguntarnos: ¿cuáles son los cambios en la forma de trabajar con el conocimiento y de qué manera la tecnología produce transformaciones en la enseñanza? ¿cómo incide el uso de tecnologías en el desempeño de los estudiantes? (UNESCO, 2017). 
Este artículo busca aportar evidencias y resultados a partir del análisis de datos secundarios de la base de datos PISA 2015 y ampliar el conocimiento acerca de la incidencia de la edad de acceso a las TIC y el uso temprano de Internet en la educación secundaria de América Latina y el Caribe (ALyC). Se estudia la correlación entre resultados de las pruebas de aprendizaje en ciencias, la edad de acceso a las TIC y el acceso temprano de los estudiantes a Internet, tanto en el hogar como en la escuela.

En ALyC es posible constatar en las últimas décadas un proceso sostenido de expansión de la oferta tecnológica gracias a las alianzas de los gobiernos de la región con sectores privados y organismos internacionales (JARA, 2015; RIVAS, 2015). El efecto visible e inmediato de ese proceso ha sido el crecimiento de la cobertura digital y la reducción de la brecha digital en el acceso a los recursos en diferentes modalidades y con programas de diferente alcance y profundidad.

Sin embargo, una revisión reciente de literatura (GALPERIN, 2017) constata que todavía persisten diferentes tipos de brechas de acceso y de resultados diferenciales de los desempeños de los estudiantes asociados al perfil social y capital cultural de la población, a los altos costos que tienen las familias para usufructuar Internet de calidad, a la ubicación geográfica de la escuela y a la escasa articulación entre las políticas tecnológicas y educativas.

La disponibilidad de información a nivel internacional y comparativa sobre el uso de las TIC en ALyC y resto del mundo es cada vez mayor. Así, la ronda 2015 del Programa Internacional de Evaluación de Estudiantes (PISA) aplicó un cuestionario para medir el grado de familiaridad con el uso de TIC de estudiantes de los países participantes en la prueba. Es a partir de la mencionada base de datos, que este artículo presenta los resultados de una investigación realizada por los autores en los siguientes países latinoamericanos y caribeños: Brasil, Chile, Colombia, Costa Rica, México, Perú, República Dominicana y Uruguay.

La investigación se propuso analizar el desempeño de los estudiantes de ALyC que participaron en PISA 2015 con respecto al comportamiento de ciertos factores o variables de uso y acceso a la tecnología consideradas de interés. En particular, el estudio busca analizar el efecto y la incidencia que tienen en los siguientes factores en los aprendizajes evaluados:

- la edad de acceso a las TIC;

- uso temprano de Internet.

Adicionalmente se ha estudiado el uso intensivo de tecnología dentro y fuera de la escuela.

Las interrogantes principales que orientan este estudio son las siguientes: 
- ¿Cuáles son los efectos de la edad de acceso a las TIC en los aprendizajes en ciencias de los jóvenes de 15 ańos participantes de PISA 2015?

- ¿Cuáles son los efectos del acceso temprano a Internet en los aprendizajes en ciencias de los jóvenes de 15 años participantes de PISA 2015?

Adicionalmente, se analiza de que manera se correlaciona el contexto (formal o informal), observado con el aprendizaje.

\section{Notas sobre el marco contextual}

Una publicación reciente de la OECD (2017) acerca de los resultados de la evaluación internacional impulsada por el programa PISA sostiene que, en prácticamente todos los países y economías evaluadas, el acceso a una computadora conectada a Internet aumentó significativamente entre los ańos 2012 y 2015. El acceso es cada vez más universal. (OECD, 2017, p.55).

La literatura sobre políticas TIC en ALyC (CLARO, 2010; CABROL; SZÉKELY, 2012) sugiere ampliar la investigación realizada, explorando nuevas variables y factores explicativos y su incidencia en la expansión y uso de la tecnología en las escuelas. Hasta ahora, la mayoría de los estudios se enfocaron en el perfil de acceso, el uso, y los efectos de las tecnologías como recursos para el aprendizaje y desarrollo de capacidades y competencias, áreas donde es posible encontrar una fuerte acumulación científica en los últimos 30 años. Sin embargo, aún queda mucho por investigar en lo que respecta a las variables individuales y las características sociales de los jóvenes y la forma en la que ellos se apropian de la tecnología.

¿Qué nos dice la investigación acerca de la exposición temprana a las TIC por parte de los estudiantes de educación secundaria? ¿Cuál es el uso que hacen los jóvenes de las TIC en el hogar y en la escuela?

La exposición temprana a Internet y el uso de TIC en los primeros años comienza a ser una realidad en ALyC. En algunos países, como en Chile y Uruguay, ya existen programas piloto de entrega de laptops a niños entre 3 y 5 años. Esta realidad es examinada por el informe McKinsey (2017), el cual evidencia que la exposición temprana a las TIC y la mayor penetración de los recursos digitales en hogares y escuelas, produce diferentes resultados.

Los datos reportados confirman que el acceso a Internet es cada vez más temprano: en los países de la OECD, el 61\% de los estudiantes informó que accedieron a Internet por primera vez cuando tenían menos de 10 años, y el 18\% informó que lo hicieron a la edad de 6 ańos o menos. El uso de Internet temprano aumentó en tres puntos porcentuales entre 2012 y 2015; en Hungría, Islandia, 
Polonia y Uruguay, el aumento fue del $7 \%$ entre aquellos estudiantes que comenzaron con seis años o menos (OECD, 2017).

Según el informe McKinsey (2017), en los países de la OCDE que participaron en la ronda 2015 de PISA, los estudiantes con exposición temprana a dispositivos digitales logran mejores resultados que el resto, pero este efecto es más pronunciado en los hogares de mayor nivel socioeconómico. El impacto de las TIC en la escuela, es mayor si los recursos son entregados por un docente que si se provee directamente a los estudiantes. Una de las conclusiones del estudio es que las TIC podrían estar contribuyendo a ampliar las brechas de inequidad.

En cuanto al uso que hacen los jóvenes de las TIC, Sunkel, Trucco y Espejo (2014) examinaron las actividades realizadas por los adolescentes de cuatro países de la región que participaron en PISA 2009 (Argentina, Brasil, Chile y Uruguay) los patrones de uso entre estos países no difieren significativamente: las principales actividades realizadas por los estudiantes de 15 años están relacionadas con usos recreativos y de comunicación, como navegar en Internet para entretenimiento, chatear, descargar música o películas y enviar mensajes de correo electrónico. Se observaron brechas de género (en algunas actividades los varones se destacan por la intensidad del juego con la computadora y actividades de ocio mientras que las mujeres prefieren hacer un uso más intenso de actividades de comunicación y uso del chat).

Recientemente, un reporte de la OECD examinó los datos de la última evaluación internacional PISA 2015 hallando que un 26\% y 16\% de los jóvenes de 15 ańos podrían ser categorizados como "usuarios extremos de Internet" ya que los fines de semana y entre semana, respectivamente, se conectan entre 4 y 6 horas por día. Brasil, Chile, Costa Rica y Uruguay integran este grupo (OECD, 2017, p. 53).

¿El uso de tecnologias y la edad de acceso son factores que inciden en el aprendizaje de competencias científicas? Reportes internacionales (RYCHEN; SALGANIK, 2003, EUROPEAN COMMISSION, 1995) definen que el estudio de la competencia cientifica de los adolecentes es relevante para las políticas educativas. Cuando los estudiantes tienen la capacidad de aprender el conocimiento de la ciencia y la tecnología, cambia la forma en que interactúan con otros y aumentan la probabilidad de usar ese conocimiento para objetivos mas amplios, como por ejemplo, incorporar una conciencia científica sobre el mundo. En tal sentido, resulta de interés conocer en qué medida en la experiencia de los jóvenes latinoamericanos participantes de este estudio, se correlaciona el aprendizaje de competencias científicas con el acceso a tecnología y el uso de Internet, aspectos claves para el desarrollo de ciudadanos competentes científicamente.

Este marco contextual que hemos descrito, es el escenario regional a partir del cual se estudian los resultados de la prueba internacional 2015 de PISA sobre educación científica de los jóvenes de 15 años de ALyC. 


\section{Metodología}

El artículo se basa en los hallazgos y resultados parciales logrados en el marco de un proyecto de investigación en el que participan los autores referido al diseño de políticas docentes efectivas que promuevan un aprendizaje profundo con el apoyo de tecnologías digitales en ALyC. Dicho proyecto busca mejorar la comprensión actual acerca de las políticas docentes, las prácticas pedagógicas y los procesos de aprendizaje basados en tecnologías digitales en los sistemas educativos del continente.

La investigación se apoya en un análisis documental comparado a partir de fuentes secundarias, bases de datos, y revisión de literatura internacional referida a los países de ALyC seleccionados para este estudio. Se analizan datos de la base pública de PISA 2015 para explorar la incidencia de la primera exposición a las TIC, el uso intensivo de las TIC en jóvenes de 15 años tanto en la escuela como en el hogar y el grado de correlación de estos factores con el aprendizaje en ciencias.

A efectos de delimitar el universo de estudio, se procedió a la depuración de la base de datos PISA 2015 en dos niveles. En la primera etapa se identificó el universo global de casos tratando de que no existieran unidades de análisis perdidas para todas las variables analizadas. Así, el tamaño de la muestra total se compone de 58.554 estudiantes para la región. En la segunda fase del análisis, tal como fuera comentado, se tomó la decisión de excluir del modelo las evidencias correspondientes a Trinidad Tobago y Argentina. El universo de estudio final se conformó con los estudiantes de los siguientes países: Brasil, Chile, Colombia, Costa Rica, México, Perú, República Dominicana y Uruguay.

Los pesos relativos de los países que integran el universo de análisis (ordenados de mayor a menor) son los siguientes: Brasil (24,4\%), Colombia (17,1\%), México (12,1\%), Chile (11,2\%), Perú (10,7\%), Costa Rica $(9,2 \%)$, Uruguay $(8,8 \%)$ y República Dominicana $(6,3 \%)$.

La Tabla 1 informa sobre la representatividad de cada país, su peso relativo al interior del tamaño de la muestra, así como la clasificación de los estudiantes según algunos indicadores estadísticos de dispersión considerados de interés. La comparación permite observar el elevado nivel de heterogeneidad de los valores de desempeño en ciencias, tanto entre países como al interior de cada uno. La dispersión relativa de los puntajes al interior de cada país es elevada, especialmente en Brasil, República Dominicana y Uruguay. A efectos de dimensionar las diferencias entre los desempeños, debe señalarse que un año de escolaridad equivale a 30 puntos en la escala de ciencias (OECD, 2016b).

El estudio recoge información cuantitativa sobre cinco variables de contexto escolar incluidas en el formulario sobre Familiaridad y uso de Tecnología de la Información y la Comunicación, uno de los dos formularios opcionales que los 
países participantes podían escoger en la ronda 2015 de PISA. De este instrumento, fueron seleccionados cuatro factores como variables independientes vinculadas a la edad y al uso de tecnología, además del indicador de los valores plausibles en ciencias (variable dependiente del modelo) a efectos de estudiar su correlación con la variable dependiente aprendizaje en ciencias.

Las variables elegidas fueron sometidas en primer lugar a un análisis descriptivo y posteriormente, luego de ser transformadas, se incluyeron en un modelo de regresión logística tipo logit.

La Tabla 2 contiene las preguntas del cuestionario analizadas en este artículo (con la codificación respectiva) e informa sobre el nivel de medición, categorías, la recodificación y las decisiones metodológicas asumidas en la transformación de las variables.

La información recogida y el posterior análisis estadístico realizado tienen la ventaja de la validez y la confiabilidad que otorgan los procedimientos de muestreo polietápico, como el llevado a cabo por PISA 2015 en cada país. Al respecto, dos limitaciones deberían considerarse. En primer lugar, las pruebas estandarizadas se basan en las declaraciones de los estudiantes y en el caso de PISA, en muchos casos los hallazgos podrían estar afectadas por la preparación específica que algunos docentes o directores realizan los días previos a su aplicación.

En segundo orden, la potencialidad y contribución del análisis estadístico a la generalización de los resultados debe entenderse a partir de los límites propios de la metodología empírica, que deliberadamente deja fuera

\section{Tabla 1}

Población de estudio, indicadores descriptivos de dispersión y media de puntaje en Ciencias, según países considerados.

\begin{tabular}{l|c|c|c|c|c|c}
\hline País & N & Mínimo & Máximo & Media* & $\begin{array}{c}\text { Desviación } \\
\text { Estándar }\end{array}$ & \% DER** \\
\hline Brasil & 14.272 & 164.90 & 718.83 & 410.57 & 82.97 & 20.2 \\
\hline Chile & 6.591 & 220.56 & 704.12 & 468.48 & 83.67 & 17.8 \\
\hline Colombia & 10.000 & 204.36 & 702.93 & 430.55 & 74.95 & 17.4 \\
\hline Costa Rica & 5.409 & 229.24 & 679.91 & 424.49 & 65.74 & 15.5 \\
\hline México & 7.110 & 227.38 & 659.74 & 423.78 & 65.30 & 15.4 \\
\hline Perú & 6.305 & 171.21 & 654.85 & 405.56 & 69.69 & 17.1 \\
\hline Rep. Dominicana & 3.700 & 156.56 & 626.84 & 344.63 & 67.92 & 19.7 \\
\hline Uruguay & 5.167 & 212.23 & 708.20 & 444.97 & 82.23 & 18.5 \\
\hline Total & 58.554 & 156.56 & 718.83 & 421.72 & 80.59 & 19.1 \\
\hline
\end{tabular}

*Promedio de las calificaciones correspondientes a 10 valores plausibles en conocimiento en ciencias; ** desvio estándar relativo; DER: desvio estándar relativo. Fuente: elaboración propia en base OECD, 2015. 
Tabla 2

Descripción de las preguntas, categorías y reformulación de las variables incluidas en el modelo.

\begin{tabular}{|c|c|c|c|c|}
\hline Código* & $\begin{array}{c}\text { Preguntas del } \\
\text { cuestionario } \\
\text { PISA 2015 } \\
\end{array}$ & $\begin{array}{l}\text { Nivel de } \\
\text { medición }\end{array}$ & Categorías & Reformulación \\
\hline $\begin{array}{l}\text { De PV1SCIE } \\
\text { a PV10SCIE }\end{array}$ & $\begin{array}{c}\text { Valores } \\
\text { plausibles en } \\
\text { Ciencia 1-10 }\end{array}$ & Escala & Numérica & $\begin{array}{c}\text { Puntaje en ciencias**: } \\
\text { Por encima de la } \\
\text { media del país }=1 \\
\text { Por debajo de la media } \\
\text { del país }=0\end{array}$ \\
\hline IC003Q01TA & $\begin{array}{l}\text { ¿Cuántos } \\
\text { años tenías la } \\
\text { primera vez } \\
\text { que usaste una } \\
\text { computadora? }\end{array}$ & Nominal & $\begin{array}{c}6 \text { años o menos } \\
\text { 7-9 años de edad } \\
\text { 10-12 años de edad } \\
13 \text { años o más viejo } \\
\begin{array}{c}\text { Nunca he usado una } \\
\text { computadora }\end{array}\end{array}$ & $\begin{array}{l}\text { Edad de acceso a las } \\
\text { TIC: } \\
6 \text { años o menos }=1 \\
\text { Resto de edades }=0\end{array}$ \\
\hline IC004Q01TA & $\begin{array}{l}\text { ¿Qué edad } \\
\text { tenías la } \\
\text { primera vez } \\
\text { que accediste a } \\
\text { Internet? }\end{array}$ & Nominal & $\begin{array}{c}6 \text { años o menos } \\
\text { 7-9 años de edad } \\
\text { 10-12 años de edad } \\
13 \text { años o más viejo } \\
\begin{array}{c}\text { Nunca he usado una } \\
\text { computadora }\end{array}\end{array}$ & $\begin{array}{l}\text { Uso temprano del } \\
\text { Internet: } \\
6 \text { años o menos = } 1 \\
\text { Resto de edades = } 0\end{array}$ \\
\hline IC005Q01TA & $\begin{array}{l}\text { Durante un } \\
\text { día de semana } \\
\text { típico, ¿por } \\
\text { cuánto tiempo } \\
\text { usas Internet } \\
\text { en tu Centro } \\
\text { educativo? }\end{array}$ & Ordinal & $\begin{array}{c}\text { Sin tiempo } \\
\text { 1-30 minutos por día } \\
\text { 31-60 minutos por día } \\
\text { 1-2 horas por día } \\
\text { 2-4 horas por día } \\
\text { 4-6 horas por día } \\
\text { Más de } 6 \text { horas por día }\end{array}$ & $\begin{array}{c}\text { Uso extremo de } \\
\text { Internet en el centro } \\
\text { educativo: } \\
\text { Más de } 4 \text { horas = } 1 \\
\text { Menos de } 4 \text { horas = } 0\end{array}$ \\
\hline IC006Q01TA & $\begin{array}{c}\text { Durante } \\
\text { un día de } \\
\text { semana típico, } \\
\text { ¿por cuánto } \\
\text { tiempo usas } \\
\text { Internet fuera } \\
\text { del Centro } \\
\text { educativo? }\end{array}$ & Ordinal & $\begin{array}{c}\text { Sin tiempo } \\
\text { 1-30 minutos por día } \\
\text { 31-60 minutos por día } \\
\text { 1-2 horas por día } \\
\text { 2-4 horas por día } \\
\text { 4-6 horas por día } \\
\text { Más de } 6 \text { horas por día }\end{array}$ & $\begin{array}{c}\text { Uso extremo de } \\
\text { Internet fuera del } \\
\text { centro educativo: } \\
\text { Más de } 4 \text { horas = } 1 \\
\text { Menos de } 4 \text { horas = } 0\end{array}$ \\
\hline
\end{tabular}

PISA: Programa Internacional para la Evaluación de Estudiantes; TIC: tecnologías de información y comunicación; *código del cuestionario escuela 2015; **es la suma de los valores plausibles en Ciencias del 1 al 10, para luego obtener el valor medio en puntaje en ciencias para cada uno de los paises. Fuente: Elaboración propia a partir de OECD, 2015. 
del modelo estadístico el mundo de las interpretaciones y representaciones sociales de los estudiantes, aspecto que todavía es escasamente explorado en los estudios internacionales.

Hecha esta salvedad, el artículo se propone analizar el rendimiento en ciencias mediante la utilización de la regresión logística, una de las principales técnicas de análisis multivariado en ciencias sociales. Los modelos de regresión logística han sido ampliamente utilizados en las ciencias sociales (CHUANG, 1997) en la investigación educativa (AUSTIN; YAFFEE; HINKLE, 1992; PENG; SO, 2002) y para el análisis de los resultados de los informes PISA (LIU; WILSON; PAEK, 2008; IBARRA; MICHALUS, 2010).

Dado que el objetivo del presente trabajo de investigación es estudiar el efecto de la edad de acceso a las TIC y el uso de Internet, así como la incidencia de uso de los dispositivos digitales dentro y fuera de la escuela en los resultados en ciencia del Informe PISA 2015, se ha optado por la utilización de modelos de regresión logística. Estos modelos, también llamados modelos de respuesta cualitativa, resultan apropiados cuando el objetivo no es predecir el comportamiento medio de un agregado, sino analizar los factores determinantes de la probabilidad dentro de un campo de acción en un grupo de opciones posibles.

Desde un punto de vista teórico, en un modelo de probabilidad no lineal, las probabilidades pueden ser acotadas en una función, F, con un rango de valores entre 0 y 1 , conforme Equación 1:

$\mathrm{P}\left(\mathrm{y}_{\mathrm{i}}=1\right)=\mathrm{F}\left(\mathrm{x}_{\mathrm{i}}^{\prime} \beta\right)$

De forma que si $\beta \mathrm{j}>0$, un incremento en $\mathrm{x}_{\mathrm{ij}}$ aumentará la probabilidad de que $y_{i}=1$. Esto significa que los coeficientes tanto positivos como negativos corresponden los efectos en misma dirección sobre la probabilidad de ocurrencia de un evento.

Donde $F\left(x_{i}^{\prime} \beta\right)$ es la función específica que garantiza que $0 \leq p \leq 1$. Así, el modelo logit se expresa de la siguiente manera (Equación 2):

$\mathrm{y}_{\mathrm{i}}=1 / 1+\mathrm{e}^{\mathrm{x} \beta}+\varepsilon_{\mathrm{i}}=\mathrm{e}^{\mathrm{xi} \beta} / 1+\mathrm{e}^{\mathrm{x} \beta}+\varepsilon_{\mathrm{i}}$

Como resultado de la estimación de los modelos logit, un coeficiente positivo aumenta la probabilidad de ocurrencia del evento, de igual forma, cuando se obtiene un parámetro con signo negativo, indica que disminuye la probabilidad de ocurrencia del evento objeto de estudio. 
Las hipótesis principales del estudio son las siguientes:

$\mathrm{H}_{1}=\mathrm{La}$ edad de acceso a las TIC a partir de los 6 años produce un efecto positivo y se correlaciona con el aprendizaje de competencias en ciencias;

$\mathrm{H}_{2}=$ El uso temprano de Internet produce un efecto positivo y se correlaciona con el aprendizaje de competencias en ciencias.

Adicionalmente se proponen dos hipótesis complementarias:

$\mathrm{H}_{3}=\mathrm{El}$ uso de Internet dentro y fuera de la escuela presenta una realidad diversa y altamente heterogénea en los estudiantes de los países analizados;

$\mathrm{H}_{4}=\mathrm{La}$ correlación entre el uso intensivo de Internet y el aprendizaje de competencias científicas depende del contexto formal (escuela) o informal (hogar).

\section{Resultados}

Los resultados se presentan en tres bloques. En primer lugar, se incluye una síntesis del análisis documental comparado con algunas evidencias de interés para la posterior discusión. Luego, la evidencia referida al abordaje descriptivo de las variables y factores considerados. Posteriormente, los hallazgos propios del modelo multivariado.

La región de ALyC, como se ha afirmado recientemente, es un "reguero de pólvora digital y se ha mantenido en un permanente movimiento de expansión tecnológica en la última década" (RIVAS, 2015, p. 89). Dicha expansión se constata en dos dimensiones que se potencian y benefician mutuamente: la infraestructura educativa y la tecnológica (JARA, 2015). En particular, la infraestructura de tecnología digital (y cada vez en mayor medida las tecnologías móviles) se expande por el continente de forma progresiva, aunque el acceso a dispositivos presenta diferencias según las condiciones geográficas (urbano, rural), edad e ingresos, entre otros factores intervinientes (SUNKEL; TRUCCO; ESPEJO, 2014).

Los datos más robustos sobre inversión pública a gran escala ponen en evidencia que las políticas TIC están más alineadas con los mercados tecnológicos que con los sistemas educativos y que, en términos generales, los responsables de políticas priorizan las decisiones sobre los dispositivos más que otras dimensiones relevantes como la pedagógica, el acceso a Internet de calidad y el soporte técnico (JARA, 2015, p. 33).

La Tabla 3 presenta algunos indicadores y datos comparados entre países para apreciar la situación en la región y el panorama en el cual se inscribe la investigación. A efectos ilustrativos, se presentan tres dimensiones consideradas de interés: modelos de infraestructural digital, cobertura de la infraestructura y desarrollo de programas emergentes en tecnologías móviles. 


\section{Tabla 3}

Indicadores sobre infraestructura digital, cobertura y cobertura. Países participantes PISA 2015.

\begin{tabular}{|c|c|c|}
\hline País & Modelo de infraestructura digital ${ }^{*}$ & $\begin{array}{l}\text { Cobertura de infraestructura } \\
\text { digital en el hogar y en las escuelas** }\end{array}$ \\
\hline Argentina & $\begin{array}{l}\text { Laboratorio y tecnología en el aula } \\
\text { (de } 1993 \text { a 2008), OLPC desde } \\
2008 \text { hasta el presente. }\end{array}$ & $\begin{array}{c}\text { 46\% de los alumnos concurren a escuelas conectadas a Internet } \\
55,5 \% \text { de los hogares tiene acceso a Internet } \\
69,4 \% \text { de la población es usuario de Internet }\end{array}$ \\
\hline Brasil & $\begin{array}{l}\text { Laboratorio y OLPC. Programa } \\
\text { ProInfo y PROUCA. }\end{array}$ & \begin{tabular}{|}
$75 \%$ de los alumnos concurren a escuelas conectadas a Internet \\
$54,5 \%$ de los hogares tienen acceso a Internet \\
$59,1 \%$ de la población es usuaria de Internet \\
\end{tabular} \\
\hline Colombia & $\begin{array}{c}\text { Laboratorio, equipamiento para las } \\
\text { escuelas desde } 1990 \text {, tecnología en el } \\
\text { aula y OLPC. }\end{array}$ & $\begin{array}{c}87 \% \text { de los alumnos concurren a escuelas conectadas a Internet } \\
42 \% \text { de los hogares tiene acceso a Internet } \\
55,9 \% \text { de la población es usuaria de Internet }\end{array}$ \\
\hline Costa Rica & $\begin{array}{l}\text { Laboratorio PRONIE (1989). } \\
\text { Conectándonos, 2011. OLPC } \\
\text { tecnologías móviles desde } 2010 .\end{array}$ & \begin{tabular}{|c|}
$81 \%$ de los alumnos concurren a escuelas conectadas a Internet \\
$60,2 \%$ de los hogares tiene acceso a Internet \\
$59,8 \%$ de la población es usuaria de Internet \\
\end{tabular} \\
\hline Chile & $\begin{array}{l}\text { Programa Enlaces, desde } 1992 . \\
\text { Laboratorios móviles computacionales, } \\
\text { tecnología en el aula y equipamiento } \\
\text { a las escuelas (2009). OLPC para } \\
\text { estudiantes de } 7^{\circ} \text { grado (2009). }\end{array}$ & $\begin{array}{l}95 \% \text { de los alumnos concurren a escuelas conectadas a Internet } \\
59,7 \% \text { de los hogares tiene acceso a Internet } \\
64,3 \% \text { de la población es usuaria de Internet }\end{array}$ \\
\hline México & $\begin{array}{l}\text { Laboratorio, proyecto Red Escolar } \\
\text { (1997), tecnologías en el aula, } \\
\text { Enciclopedia y OLPC desde } 2013 .\end{array}$ & $\begin{array}{c}44 \% \text { de los alumnos concurren a escuelas conectadas a Internet } \\
\text { 39\% de los hogares tiene acceso a Internet } \\
57,4 \% \text { de la población es usuaria de Internet } \\
\end{array}$ \\
\hline Perú & $\begin{array}{l}\text { Laboratorio y OLPC. Programa } \\
\text { Huascarán } 2008 \text { (inicial y primaria y } \\
\text { secundaria: } 900.000 \text { laptops). }\end{array}$ & $\begin{array}{c}66 \% \text { de los alumnos concurren a escuelas conectadas a Internet } \\
23,3 \% \text { de los hogares tiene acceso a Internet } \\
40,9 \% \text { de la población es usuaria de Internet }\end{array}$ \\
\hline Uruguay & $\begin{array}{l}\text { Laboratorios digitales, LABTED y } \\
\text { OLPC. CEIBAL (2006) (primaria y } \\
\text { secundaria: } 1.100 .100 \text { laptops). }\end{array}$ & \begin{tabular}{|c|}
$76 \%$ de los alumnos concurren a escuelas conectadas a Internet \\
$59,7 \%$ de los hogares tiene acceso a Internet \\
$64,6 \%$ de la población es usuaria de Internet \\
\end{tabular} \\
\hline $\begin{array}{l}\text { República } \\
\text { Dominicana }\end{array}$ & $\begin{array}{l}\text { Programa de Informática PIE } \\
\quad \text { (laboratorios desde 1996), } \\
\text { Compumaestro: } 22.000 \text { paquetes } \\
\text { tecnológicos entregados en } 2004 .\end{array}$ & $\begin{array}{l}26 \% \text { de los alumnos concurren a escuelas conectadas a Internet } \\
\begin{array}{l}23,6 \% \text { de los hogares tiene acceso a Internet } \\
54,2 \% \text { de la población es usuaria de Internet }\end{array}\end{array}$ \\
\hline
\end{tabular}

Fuente: *Los modelos, basados en Jara (2015), se refieren al énfasis en el equipamiento del Laboratorio o sala de Informática, la tecnología en el aula o los programas universales uno a uno; **Banco de Datos de CEPAL, Cepalstat, última información reportada del 2015, disponible en: <http://estadisticas.cepal.orgl cepalstat/web_cepalstatlestadisticasindicadores.asp $>$.

OLPC: One Laptop per Child; ProInfo: Programa Nacional de Tecnologia Educacional; PROUCA:

Programa Um Computador por Aluno; PRONIE: Programa Nacional de Incentivo à Educação Escolar Básica Gratuita; LABTEC: laboratorios tecnológicos; PIE: Programa de Informática Educativa. 
Las evidencias recopiladas en la Tabla 3, ilustran sobre los programas de inversión pública y políticas TIC implementados en la últimas dos décadas, la multiplicidad de modelos adoptados (en la mayoría de los casos yuxtapuestos con énfasis en políticas OLPC), la diversidad en el acceso a Internet dentro y fuera de la escuela y la incorporación reciente en todos los casos analizados de múltiples iniciativas en el marco de programas educativos basados en las tecnologías móviles y en dispositivos

Los resultados evidencian que hay una importante expansión tecnológica en términos de infraestructura digital a región de ALyC y que existe una gran diversidad en los sistemas educativos de modelos y programas. Sin embargo, ello no necesariamente ha tenido incidencia en los aprendizajes en ciencias tal como veremos en los apartados que siguen.

\section{Edad de acceso a TIC y uso temprano a Internet de los países de Aly C participantes de PISA 2105}

En esta sección se presenta un análisis descriptivo de las variables consideradas en el estudio. Tal como se puede apreciar en el Gráfico 1, la disposición a usar tecnología de manera intensiva en el hogar o fuera de la escuela, es entre tres y cuatro veces superior que el uso en los centros educativos de los países participantes de PISA 2015. El promedio de uso dentro de la escuela es similar en la región de ALyC y $\operatorname{OCDE}(9,1 \%$ y $10 \%)$. Los estudiantes latinoamericanos tienen un uso intensivo por fuera de las instituciones más acentuado que el promedio de OCDE. Tanto en el uso en la escuela, como fuera de ella, los alumnos de México, Perú y República Dominicana acceden a Internet en un porcentaje menor que la media de la región. En los países con mayor tradición de políticas TIC del continente, como Brasil, Chile, Colombia, Costa Rica y Uruguay los estudiantes secundarios manifiestan una alta predisposición al uso intensivo de tecnologías fuera de los centros educativos.

En el Gráfico 2 se puede observar la densidad del acceso temprano a Internet y la intensidad del uso dentro y fuera de los centros educativos evaluados. Nuevamente se constata un escenario heterogéneo para la región. Con excepción de México y Perú, países con políticas TIC incipientes y en desarrollo, el resto de los países tienen una elevada tasa de acceso temprano (superior al promedio de OCDE). Intuitivamente, los datos observados parecerían corroborar la hipótesis de correlación entre la edad de acceso temprano (6 años de edad), con el uso intensivo en el marco de las políticas OLPC del continente.

Finalmente, en el Gráfico 3, se puede constatar cierta correlación entre los países con ventaja relativa con respecto al uso y acceso temprano (Chile, Costa Rica y Uruguay), en comparación con el resto de los sistemas educativos considerados. La distribución de los pesos relativos, tanto en el acceso a las TIC como al uso temprano de Internet, presenta una distribución similar en los países analizados, y pone de manifiesto especialmente dos aspectos: la expan- 
sión secuencial de la tecnología (primero el acceso al computador sin Internet y posteriormente a la conexión) y la heterogeneidad observada entre países con realidades muy diferentes (donde México y Perú se destacan como países ubicados por debajo del promedio regional con respecto a las variables analizadas).

\section{Análisis del modelo de regresión logística}

Los resultados y estimaciones obtenidas para el modelo multivariado se presentan en la Tabla 4.

Los hallazgos y las interpretaciones estadísticas se presentan en función de las cuatro variables independientes incluidas en el modelo.

\section{Edad de acceso a las TIC}

En primer lugar, el examen de los datos corrobora que los estudiantes que comienzan a usar la computadora con 6 años o menos tienen mayor probabi-

\section{Gráfico 1}

Usuarios extremos de Internet dentro y fuera de la escuela, según países y regiones consideradas.

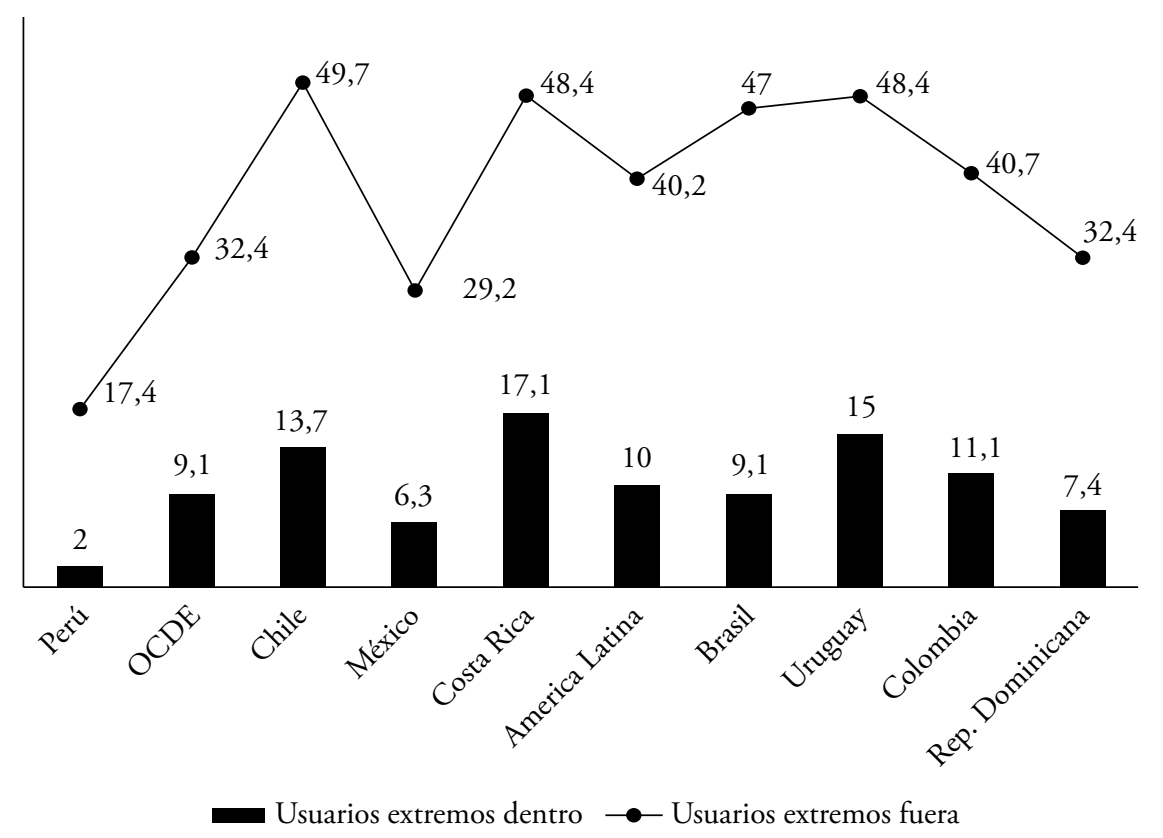

OECD: Organisation for Economic Co-Operation and Development. Indicador: proporción de alumnos que usan Internet más de 4 horas diarias dentro y fuera de la escuela.

Fuente: elaboración propia en base a datos OECD, 2015. 
lidad de conseguir un puntaje en ciencias por encima de la media de la población considerada. La evidencia es robusta y consistente para toda la región (el resultado es significativo para todos los países incluidos en este informe, en todos los casos con valores de significatividad del 1\%). De manera más específica, los jóvenes de 15 años que se inician temprano con el uso de una computadora tienen una probabilidad dos veces superior de obtener un puntaje en ciencias por encima de la media que el resto del estudiantado (véase Anexo 1). Los resultados son coincidentes con los encontrados en el Informe McKinsey (2017) quienes detectan que los aprendizajes aumentan un $20 \%$ en promedio, cuando los alumnos comienzan a usar tempranamente las computadoras. En la misma línea, Fernández-Aráuz (2014) demostró para Costa Rica, con datos de PISA 2012, que la edad temprana de acceso a las TIC es un factor que determina el rendimiento en matemática de los estudiantes. El acceso temprano en la escuela debe estar acompañado, tal como fuera seńalado por Buckingham (2008) por un rol activo de la familia y por una fuerte predisposición a usar en el hogar el lenguaje digital. ¿¿Cuáles son los efectos que produce el acceso temprano a las TIC en la adquisición de competencias científicas? Investigaciones previas señalan la contribución de la introducción temprana de tecnología en la educación infantil como el desarrollo de inteligencias múltiples, alfabetización tecnológica en entornos virtuales y la facilitación de la globalización del conocimiento científico para un aprendizaje significativo (AGUIAR-PERERA;

\section{Gráfico 2}

Porcentaje de jóvenes de América Latina y Caribe que accedieron de forma temprana a una computadora y hacen un uso extremo dentro y fuera la escuela según países participantes de PISA 2015*.

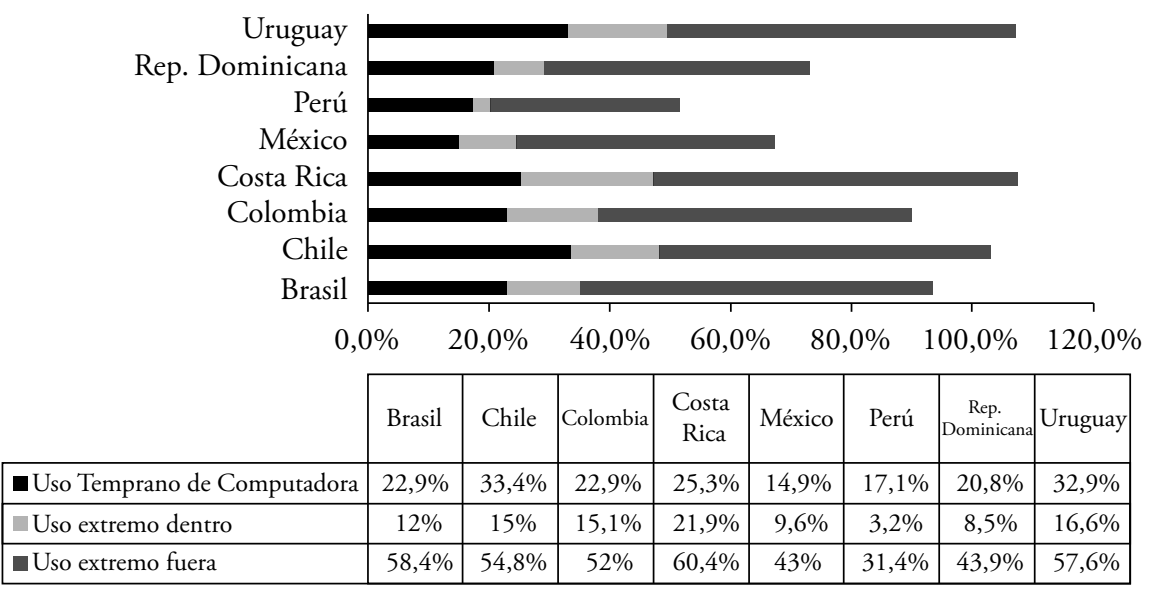

*El indicador es el porcentaje de jóvenes de 6 años que hacen un uso temprano de la computadora. Los porcentajes de uso extremo dentro y fuera se calculan sobre el total de alumnos que comenzaron a usar la computadora con 6 años.

Fuente: elaboración propia en base a datos OECD, 2015. 
CUESTA-SUÁREZ, 2009; BUCKINGHAM, 2008). La ventaja del uso de tecnología digital a temprana edad resulta en un mayor interés y participación de los niños y niñas en el desarrollo de habilidades y competencias en educación infantil (CASCALES-MARTÍNEZ; LAGUNA SAVOIA, 2014).

\section{Uso temprano de Internet}

En cambio, la incidencia del uso temprano de Internet no parece tener efectos tan lineales y directos. Por ejemplo, el hecho de comenzar a usar Internet con 6 años o menos de edad tiene un efecto negativo y significativo sobre el puntaje en ciencias en Colombia, México y Uruguay (con valores de los coeficientes: -0,391, $-0,327$ y - $-0,361$, respectivamente). Chile se destaca en la región por ser el único país que presenta una relación positiva y significativa con respecto a la correlación entre el puntaje en ciencia y acceso temprano a los recursos digitales e Internet $(0,178)$. Es muy probable que la navegación temprana este asociado, como lo han demostrado estudios recientes, con el uso recreativo del dispositivo, y que, en consecuencia, aporte muy poco al rendimiento de los estudiantes. El uso recreativo, en promedio, tiene efectos negativos en los desempeños de los estudiantes (UNESCO, 2016, p. 4).

\section{Gráfico 3}

Porcentaje de jóvenes de 15 años de América Latina y el Caribe según uso temprano de computadora y de Internet. Países participantes PISA 2015*.

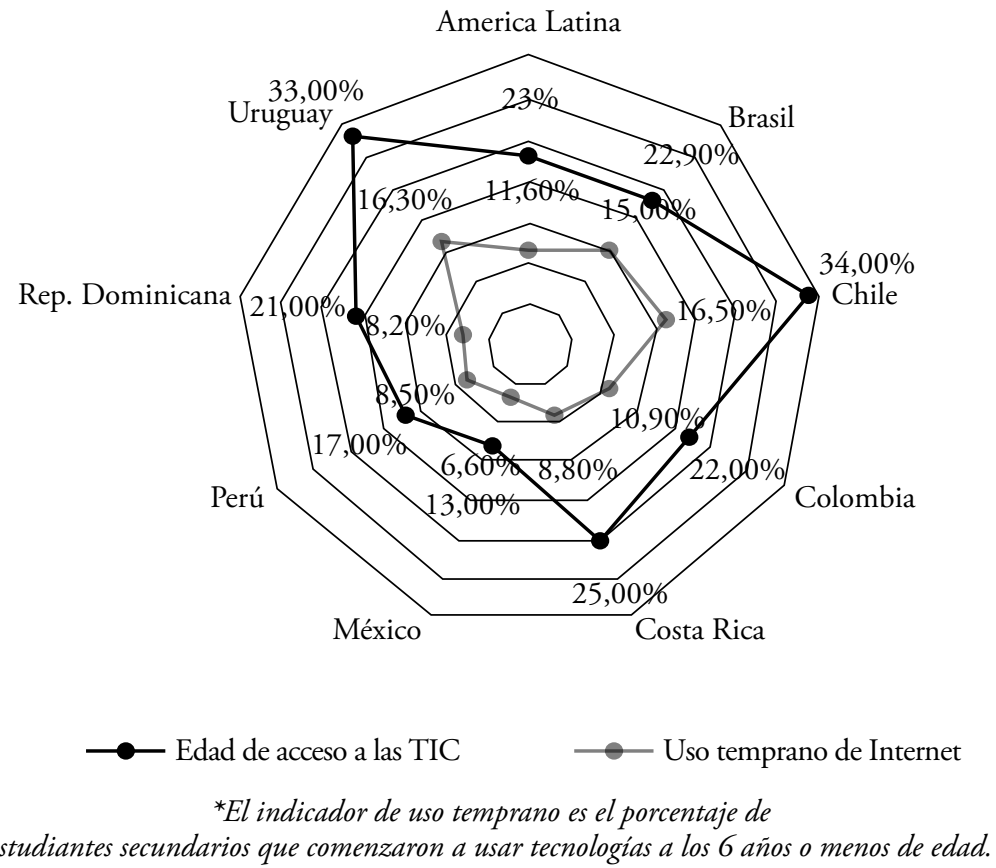




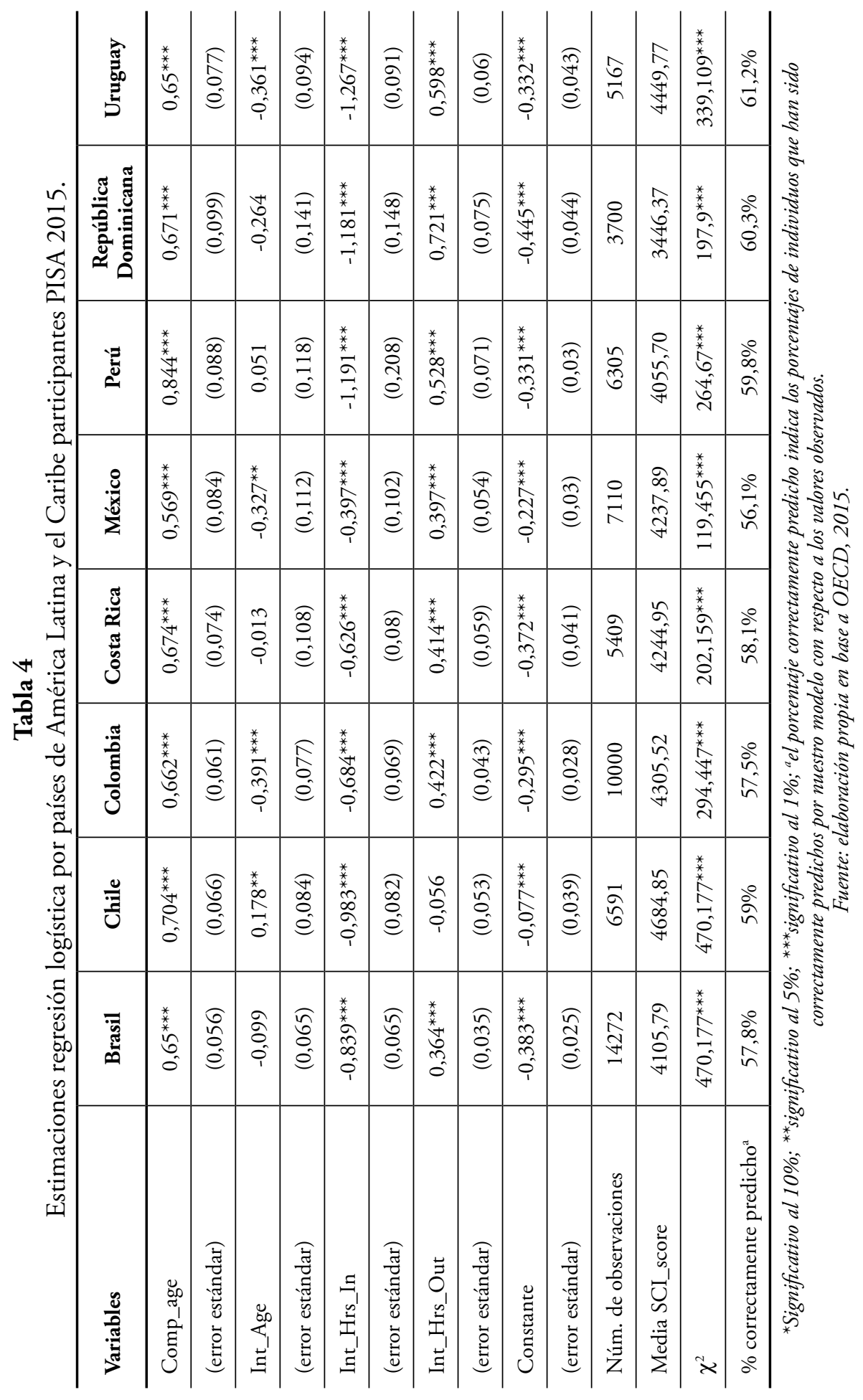


Para los casos de Brasil, Costa Rica, Perú y República Dominicana, los resultados evidencian una relación negativa, pero dicho coeficiente no resultó ser estadísticamente significativo. En síntesis, los países analizados en este documento presentan un nivel elevado de heterogeneidad, similar a los hallados en la literatura internacional sobre el tema. En Chile se combinan políticas de acceso universal a las tecnologías digitales con estrategias contextualizadas en las escuelas, como los laboratorios (JARA, 2015). Este hecho podría explicar los resultados diferenciales observados en este país y podría ser una pista para explorar en investigaciones futuras.

\section{Uso intensivo de Internet en el centro educativo}

¿Cuánto aporta el uso intensivo de Internet en las instituciones educativas al aprendizaje en ciencias de los estudiantes de Latinoamérica?

Las evidencias recogidas sostienen la hipótesis de que la relación es inversa: a mayor intensidad del uso, menor aprendizaje. En efecto, navegar y hacer uso intensivo de Internet en el horario escolar (en promedio 4 horas por día, incluyendo horarios de clase y tiempo extra clase de la jornada escolar) tiene un efecto negativo y significativo sobre el puntaje en ciencias en PISA para todos los países de América Latina incluidos en el modelo. La sobre estimulación del uso intensivo de la tecnología y la escasa intervención pedagógica de los docentes en estos casos, introduce factores distractores que desalientan los efectos positivos esperados (MCKINSEY, 2017). Si bien es cierto que todavía pocos estudios han explorado en profunidad qué problemas pueden ocurrir como consecuencia del uso intensivo de la tecnología, a nivel descriptivo algunas evidencias indican que los aspectos negativos refieren al escaso desarrollo de habilidades emocionales, el aumento de la dependencia digital y la adicción a la pantalla que afectarían, en parte, el bienestar mental de los niños y niñas (UNICEF, 2017). El uso moderado (algunas veces a la semana) con direccionamiento pedagógico son los factores claves (ARIAS; CRISTIA, 2014).

Entre países, se constatan algunas diferencias. Brasil, Chile y Colombia tienen una probabilidad dos veces menor de obtener un puntaje en ciencias por encima de la media. En cambio, en Perú, República Dominicana y Uruguay, es hasta tres veces menos dicha probabilidad (véase Anexo 1). En tal sentido, tal como lo ha demostrado el informe McKinsey, el impacto de la exposición digital es variable. Algunas hipótesis estarían indicando que, si los recursos tecnológicos son entregados y supervisados por los docentes, las TIC tienen efectos más beneficiosos. El acceso irrestricto y sin planificación docente a ciertas tecnologías como tabletas y lectores electrónicos, produce efectos perjudiciales para el aprendizaje (MCKINSEY, 2017, p. 35, gráfico 22). Todo parece indicar que el desafío es alinear la formación de los profesores en el uso pedagógico de las TIC (VAILLANT; RODRÍGUEZ ZIDÁN; BERNASCONI, 2015) con el uso selectivo y focalizado de recursos digitales para actividades específicas enmarcadas en el currículum escolar. 


\section{Uso intensivo de Internet fuera del centro educativo}

El uso intensivo de Internet en el hogar o fuera de los centros de estudio fue incluido como un nuevo factor explicativo. El hallazgo más significativo es el hecho de que el uso intensivo de Internet fuera del centro educativo tiene un efecto positivo sobre el puntaje en ciencias en PISA 2015. Este resultado es significativo para todos los países de América Latina a excepción de Chile, donde el coeficiente de la variable posee un signo negativo $(-0,056)$, sin embargo, dicho coeficiente no resultó ser significativo. Los resultados confirman los hallazgos de Spiezia (2010) realizado en base a PISA 2006, Alderete y Formichella (2016) que aplicaron un modelo multinivel para PISA 2012 y Notten $e t$ al. (2009). Los tres estudios precedentes, son consistentes en identificar como factor causal predominante el uso educativo de herramientas tecnológicas en el hogar como elemento positivo asociado a los mejores resultados en ciencias, con un peso relativo superior a la incidencia de las TIC utilizadas en el aula.

Por último, los resultados presentados en este artículo merecen ser analizados teniendo en cuenta el contexto y otros factores que correlacionan con el aprendizaje de la ciencias de los jóvenes de 15 años. La relación entre el estatus socioeconómico y el rendimiento en los países latinoamericanos examinados, es tan sólida como la media de los países de la OCDE y, en el caso de Chile, Perú y Uruguay, significativamente más potente. Esa relación entre estatus socioeconómico y rendimiento implica que las posibilidades de lograr buenos resultados siguen siendo relativamente bajas en el caso de los alumnos desfavorecidos, en comparación con sus pares provenientes de contextos más favorecidos.

Comparar la relación entre la ventaja socioeconómica y el rendimiento alto y bajo indica además, que en todos los países de América Latina, excepto Chile, la inclinación en la relación en el caso del rendimiento elevado es superior a la que se da con un rendimiento bajo. Parecería que el estatus socioeconómico reduce las posibilidades de los alumnos desfavorecidos de alcanzar niveles de rendimiento elevados y en mayor medida que protege a los alumnos favorecidos de niveles de rendimiento relativamente bajos (OECD, 2016a, tabla I.6.5).

Sin embargo, las posibilidades de que los alumnos alcancen un nivel básico de rendimiento en ciencias, lectura o matemáticas ${ }^{2}$ son en general mucho menores para los alumnos desfavorecidos que para el resto. En la Figura 2.10 se comparan las probabilidades de alcanzar un nivel básico de rendimiento del 25\% de los alumnos con el estatus socioeconómico más bajo en cada país con las probabilidades del 75\% restante de los alumnos.

En la República Dominicana (donde solo una pequeña minoría de alumnos alcanza el nivel básico en diversas materias), el 25\% constituido por los alumnos más desfavorecidos tienen más de seis veces más probabilidades de rendir por debajo del nivel básico en todas las materias. En Perú, los alumnos des- 
favorecidos tienen unas 15 veces más probabilidades que el resto de los estudiantes de rendir en los niveles más bajos de competencia en lectura, y corren asimismo un riesgo desproporcionado de bajo rendimiento en las restantes áreas temáticas.

Guadalupe y Villanueva (2013) analizaron el desempeño de la competencia lectora y su relación con las características socioeconómicas para cinco países de América Latina (Argentina, Brasil, Chile, México y Perú) y concluyeron que existe una clara correlación. Sin embargo, destacan que el diseño de PISA no permite medir trayectorias educativas explicado por variables distintas al nivel socioeconómico, es decir

que los puntajes en 2009 sean mayores que en 2000 no quiere decir, necesariamente, que el sistema educativo de 2009 sea mejor (en la misma proporción) que el de 2000, ya que los efectos de la operación de los sistemas educativos se producen en plazos temporales prolongados y concurriendo con una gran diversidad de fenómenos sociales (GUADALUPE; VILLANUEVA, 2013, p. 178).

Podemos concluir que la explicación de las diferencias en los desempeños evaluados por PISA es de tipo multicausal y obedece a multiples factores. El estudio de Castro-Aristizabal, Castillo-Caicedo y Mendoza-Parra (2017), encontró que la variabilidad de los desempeños en las competencias evaluadas por PISA es atribuible en menor medida a la heterogenidad de las caracteristicas de los estudiantes que a otros factores contextuales escolares (contexto público o privado del centro, calidad de los materiales curriculares).

\section{Discusión y conclusiones}

Los datos analizados en este artículo exponen con claridad el énfasis dado en las políticas TIC de ALyC en los aspectos vinculados a la infraestructura, la conectividad, los modelos tecnológicos desarrollados y más recientemente, el incremento de Internet móvil. No obstante, en la misma línea que en otros estudios comparados (GALPERIN, 2017), el crecimiento observado de la inversión en TIC es acompañado por procesos que producen una fuerte inequidad digital y brechas entre diferentes tipos de usuarios de Internet. Hay diversos estudios que coinciden con este hallazgo, que no solo refiere a la región sino al resto de países de la OCDE (BECTA, 2004; HARGITTAI; HSIEH, 2005).

En segundo lugar, las hipótesis examinadas respecto a la relación entre uso temprano de las TIC y de Internet, uso intensivo dentro y fuera de la escuela con el aprendizaje en ciencias, revelan una realidad compleja y multidimensional, con diferentes efectos de las TIC sobre los resultados académicos. 
Usar la computadora tempranamente, tiene una correlación positiva y significativa con el aprendizaje en todos los países de la región incluidos en el estudio. Sin embargo, no basta con la promoción del uso temprano de Internet para incidir en los resultados educativos, siendo este uno de los desafíos mayores que las políticas educativas deberían encarar. ¿El uso intensivo de la navegación por la web tiene efectos diferentes en los logros educativos de los estudiantes, según se realice en la escuela o en el hogar?

Uno de los hallazgos de este estudio es haber identificado efectos independientes de las TIC en función del contexto de uso. Es muy probable que esto ocurra debido al elevado nivel de disparidad no solo de los recursos entre las escuelas de la región de América Latina y Caribe, — tanto a nivel nacional como entre países - (OCDE, 2015) sino en la forma en que las escuelas y los docentes utilizan la tecnología. Diversos reportes muestran que los profesores, igual que los estudiantes, prefieren usar con mayor frecuencia las TIC en su hogar y no en la sala de clases (HINOSTROZA et al., 2015). Las evidencias analizadas muestran que el uso extremo de recursos tecnológicos es casi cuatro veces mayor en el hogar que en la escuela, pero sus impactos son muy diferentes. En la escuela tiene un efecto adverso sobre el aprendizaje, lo que pone al docente, tal como otros estudios ya han demostrado, como el actor fundamental para direccionar el cambio educativo con apoyo de tecnologías digitales (FULLAN; LANGWORTHY, 2013). Esta idea es apoyada por los estudios que plantean como urgente la necesidad de definir estándares de uso de TIC en la formación docente (HINOSTROZA, 2017).

Un nuevo aspecto a resaltar -a ser profundizado en futuros estudios-, es la contribución positiva del uso intensivo de las TIC en el hogar con respecto al aprendizaje de cometencias científicas.

Probablemente uno de los factores que explican este fenómeno es el desarrollo constante de aplicaciones y software de apoyo para la enseñanza de las ciencias. Un tópico a profundizar en nuevos estudios es conocer las experiencias de aprendizajes de los estudiantes en contextos informales y preguntarse de qué manera incide el capital tecnológico (saberes, competencias y recursos de las familias) en este proceso (HOWARD, 1992).

Esta hipótesis debería ser ampliada y analizada con nuevos estudios multivariados que controlen las variables de contexto socioeconómico y las características individuales de los estudiantes (género, desempeño académico del estudiante), con el objetivo de disponer de nuevas evidencias empíricas y más insumos para apoyar a las docentes de la región.

Considerando este último aspecto, surge como desafío diseñar estrategias institucionales para articular el uso educativo de las herramientas tecnológicas entre el hogar y la escuela. La conexión entre los diferentes espacios de socialización (formales e informales) es indispensable para potenciar los efectos positivos de las TIC.

Tal como fuera demostrado en este estudio, además de las brechas en el acceso y en los diferentes patrones de uso de las TIC (DIMAGGIO; HARGITTAI, 2001) esta- 
mos frente a una nueva manifestación de la desigualdad digital expresada en las brechas en los resultados académicos (RIGGINS; DEWAN, 2009). Los datos examinados en este informe muestran un panorama de expansión tecnológica constante, pero a la vez una realidad compleja y desafiante para los sistemas educativos de la región.

En base a los hallazgos comentados, algunas de las pistas que deberán ser contrastadas y ampliadas con nuevas investigaciones en ALyC refieren a la necesidad de promover políticas de uso educativo de los recursos con equidad digital, potenciar la apropiación pedagógica de las tecnologías con programas y proyectos integrales y hacer un seguimiento y monitoreo de la inversión en infraestructura digital para asegurarnos que efectivamente esté al servicio de una enseñanza y aprendizaje de calidad para todos.

\section{Notas}

1. En este informe no figura Argentina entre los países analizados porque por decisión de la OCDE el país quedó excluido ya que la muestra no cubría la población objetivo. Tampoco aparece Trinidad Tobago, país que se excluyó por la escasez de casos válidos y de homogeneidad en las variables de estudio.

2. Es importante recalcar que las pruebas PISA, se llevan a cabo cada 3 años y evaluan tres áreas de competencias: lectura, matemáticas y ciencias, donde a cada ciclo se centra en una de las tres áreas temáticas. Así, este ciclo de evaluaciones se resume de la siguiente manera: ańo 2000 (lectura), año 2003 (matemáticas), año 2006 (ciencias), año 2009 (lectura), año 2012 (matemáticas) y el año 2015 (ciencias).

\section{Referencias}

AGUIAR-PERERA, M. V.; CUESTA-SUÁREZ, H. Importancia de trabajar las TIC en educación infantil a través de métodos como la webquest. PIXEL BIT: Revista de Medios y Educación, n. 34, p.81-94, 2009.

ALDERETE, V.; FORMICHELLA, M. Efecto de las TIC en el rendimiento educativo: el Programa Conectar Igualdad en la Argentina. Revista CEPAL, n. 119, p. 89-107, 2016.

ARIAS, E.; CRISTIA, J. El BID y la tecnología para mejorar el aprendizaje: ¿cómo promover programas efectivos? 2014. Disponible en: < https://publications.iadb.org/en/ publication/17450/idb-and-technology-education-how-promote-effective-programs $>$. Acceso en: 2 dic. 2018.

AUSTIN, J. T.; YAFFEE, R. A.; HINKLE, D. E. Logistic regression for research in higher education. In: SMART, J. C. (ed.). Higher Education: Handbook of Theory and Research. Nova York: Springer, 1992. v. 8. p. 379-410.

BRITISH EDUCATIONAL COMMUNICATIONS AND TECHNOLOGY AGENCY (BECTA). A review of the research literature on barriers to the uptake of ICT by teachers. Reino Unido: British Educational Communications and Technology 
Agency, 2004. Disponível em: <http://partners.becta.org.uk/uploaddir/downloads/page documents/research/barriers.pdf $>$. Acesso em: 13 maio 2018.

BUCKINGHAM, D. Más allá de la tecnología: aprendizaje infantil en la era digital. Buenos Aires: Manantial, 2008.

CABROL, M.; SZÉKELY, M. (eds.). Educación para la transformación. Washington, D. C.: BID, 2012. Disponível em: <https://publications.iadb.org/es/publicacion/14245/ educacion-para-la-transformacion>. Acesso em: 13 maio 2018.

CASCALES-MARTÍNEZ, A.; LAGUNA SAVOIA, I. Una experiencia de aprendizaje con la pizarra digital interactiva en educación infantil. PIXEL BIT: Revista de Medios y Educación, n. 45, p. 125-136, 2014. https://doi.org/10.12795/pixelbit.2014.i45.09

CASTRO-ARISTIZABAL, G.; CASTILLO-CAICEDO, M.; MENDOZA-PARRA, J. Factores asociados a la adquisición de competencias en América Latina. Revista de Ciencias Sociales, v. 23, n. 4, p. 33-52, 2017.

CHUANG, H. L. High school youth's dropout and re-enrollment behavior. Economics of Education Review, v. 16, n. 2, p. 171-186, 1997. https://doi.org/10.1016/S02727757(96)00058-1

CLARO, M. Impacto de las TIC en los aprendizajes de los estudiantes. Estado del arte. CEPAL, 2010. 28 p. (Colección Documentos de Proyectos, n. 339).

DIMAGGIO, P.; HARGITTAI, E. From the "Digital Divide" to "Digital Inequality": Studying Internet use as penetration increase. Nova Jersey: Center for Arts and Cultural Policy Studies, Princeton University, 2001. 25 p. (Working Paper, n. 15).

EUROPEAN COMMISSION. White paper on education and training: Teaching and learning. Towards the learning society. Luxembourg: Office for Official Publications in European Countries, 1995.

FERNÁNDEZ-ARÁUZ, A. La influencia del acceso al uso de computadoras en edad temprana sobre el rendimiento en matemáticas. Ciencias Económicas, v. 32 n. 2, p. 113-125, 2014.

FONDO DE LAS NACIONES UNIDAS PARA LA INFANCIA (UNICEF). Estado Mundial de la Infancia 2017: Niños en un mundo digital. Nueva York: UNICEF, 2017.

FULLAN, M.; LANGWORTHY, M. Towards a new end: new pedagogies for deep learning. Seattle: Collaborative Impact, 2013. Disponível em: $<$ http://www.newpedagogies. nl/images/towards a new end.pdf>. Acesso em: 13 maio 2018.

GALPERIN, H. Digital society: Gaps and challenges for digital inclusion in Latin America and the Caribbean. Policy Papers UNESCO. Montevideo: UNESCO, 2017.

GUADALUPE, C.; VILLANUEVA, A. PISA 2009/2000 en América Latina: una relectura de los cambios en el desempeño lector y su relación con las condiciones sociales. Apuntes. Revista de Ciencias Sociales, v. 40, n. 72, p. 157-192, 2013. https://doi.org/10.21678/ apuntes. 72.678

HARGITTAI, E.; HSIEH, Y. P. Digital Inequality. In: DUTTON, W. H. (ed.). The Oxford Handbook of Internet Studies. Oxford: Oxford University Press, 2013. p. 129-150. 
HINOSTROZA, J. E. ICT, education and social development in Latin America and the Caribbean. Montevideo: UNESCO Institute for Statistics, 2017. Disponível em: $<$ https://cetic.br/publicacao/ict-education-and-social-development-in-latin-america-andthe-caribbean/>. Acesso em: 13 maio 2018.

HINOSTROZA, J. E.; IBIETA, A.; CLARO, M.; LABBÉ, C. Characterisation of teachers' use of computers and Internet inside and outside the classroom: The need to focus on the quality. Education and Information Technologies, v. 21, n. 6, p. 15951610, 2015. http://dx.doi.org/10.1007/s10639-015-9404-6

HOWARD, T. Connectivity, and Computer Literacy. Computers and Composition, v. 9, n. 3, 41-58, 1992.

IBARRA, M. C.; MICHALUS, J. C. Análisis del rendimiento académico mediante un modelo logit. Ingeniería Industrial, v. 9, n. 2, p. 47-56, 2010.

JARA, I. Infraestructura digital en educación: Avances y desafíos para América Latina. UNESCO, 2015. (Cuaderno SITEAL). Disponível em: <https://documentos. tips/download/cuaderno-infraestructura-digital-para-educacion-avances-y-desafios-paralatinoamerica 5a0fe4e9d64ab246defa05f4 pdf>. Acesso em: 13 maio 2018.

LIU, O. L.; WILSON, M.; PAEK, I. A multidimensional Rasch analysis of gender differences in PISA mathematics. Journal of Applied Measurement, v. 9, n. 1, p. 18-35, 2008.

MCKINSEY. Factores que inciden en el desempeño de los estudiantes: perspectivas de América Latina. Educación. Nova York: McKinsey \& Company, 2017.

NOTTEN, N.; PETER, J.; KRAAYKAMP, G.; VALKENBURG, P. M. Digital divide across borders: A crossnational study of adolescents' use of digital technologies. European Sociological Review, v. 25, n. 5, p. 551-560, 2009. https://doi.org/10.1093/esr/jen071

ORGANIZACIÓN DE LAS NACIONES UNIDAS PARA LA EDUCACIÓN, LA CIENCIA Y LA CULTURA (UNESCO). Terce en la mira: uso recreativo del computador: cuánto aporta al rendimiento de los estudiantes? Santiago: UNESCO/ OREALC, 2016. v. 2.

ORGANIZACIÓN DE LAS NACIONES UNIDAS PARA LA EDUCACIÓN, LA CIENCIA Y LA CULTURA (UNESCO). E2030: Education and skills for the 21st century. Regional Meeting of Ministers of Education of Latin America and the Caribbean, Buenos Aires, Argentina, January 24-25, 2017. Santiago: UNESCO, 2017.

ORGANISATION FOR ECONOMIC CO-OPERATION AND DEVELOPMENT (OECD). PISA 2015 Results Excellence and Equity in Education. Paris: Organisation for Economic Co-operation and Development (OECD), 2016a. v. 1.

ORGANISATION FOR ECONOMIC CO-OPERATION AND DEVELOPMENT (OECD). PISA 2015 Resultados Clave. Paris: Organisation for Economic Co-operation and Development (OECD), 2016b.

ORGANISATION FOR ECONOMIC CO-OPERATION AND DEVELOPMENT (OECD). PISA 2015 Results Students' well-being PISA. Paris: Organisation for Economic Co-operation and Development (OECD), 2017. v. 3. 
ORGANISATION FOR ECONOMIC CO-OPERATION AND DEVELOPMENT (OECD). Students, Computers and Learning: Making the connection PISA. Paris: Organisation for Economic Co-operation and Development (OECD), 2015.

PENG, C. Y.; SO, T. S. H. Modelling strategies in logistic regression with SAS, SPSS, Systat, BMDP, Minitab, and STATA. Journal of Modern Applied Statistical Methods, v. 1, n. 1, p. 147-156, 2002. https://doi.org/10.22237/jmasm/1020255720

RIGGINS, F.; DEWAN, S. The digital divide: Current and future research directions. Journal of the Association for Information Systems, v. 6, n. 12, p. 298-337, 2009. https://doi.org/10.17705/1jais.00074

RIVAS, A. Latin America after PISA: Lessons learned about education in seven countries. Executive Summary. Buenos Aires: CIPPEC-Natura-Instituto Natura, 2015.

RYCHEN, D. S.; SALGANIK, L. H. (eds.). Definition and Selection of Key competencies: Executive Summary. Göttingen, Germany: Hogrefe, 2003.

SPIEZIA, V. Does computer use increase educational achievements? Student-level evidence from PISA. OECD Journal: Economic Studies, v. 2010, n. 1, p. 1-22, 2010. Disponível em: <http://www1.oecd.org/eco/labour/49849896.pdf>. Acesso em: 13 maio 2018.

SUNKEL, D.; TRUCCO, M.; ESPEJO, A. La integración de las tecnologías digitales en las escuelas de América Latina y el Caribe: una mirada multidimensional. Chile: CEPAL, 2014.

VAILLANT, D., RODRÍGUEZ ZIDÁN, E. Y BERNASCONI, G. En qué cambian las prácticas de enseńanza de la matemática en un "modelo 1:1" a escala nacional. Revista Complutense de Educación, v. 26, n. 2, 2015.

\section{SOBRE LOS AUTORES}

Denise Vaillant es directora del Programa de Doctorado en Educación del Instituto de Educación de la Universidad ORT Uruguay. Investigadora Nivel II del Sistema Nacional de Investigadores del Uruguay.

EduARdo RodrígueZ-Zidán es profesor e investigador del Instituto de Educación de la Universidad ORT Uruguay. Investigador Nivel I del Sistema Nacional de Investigadores del Uruguay.

Juan Pablo Zorrilla-Salgador es profesor e investigador de Economía y Finanzas de la Facultad de Administración y Ciencias Sociales de la Universidad ORT Uruguay.

Recibido en el 15 de mayo de 2018.

Aceptado en el 31 de enero de 2019. 


\section{Anexo 1}

Odds ratios por países de América Latina.

\begin{tabular}{|c|c|c|c|c|c|c|c|c|}
\hline Variables & Brasil & Chile & Colombia & $\begin{array}{l}\text { Costa } \\
\text { Rica }\end{array}$ & México & Perú & $\begin{array}{c}\text { República } \\
\text { Dominicana }\end{array}$ & Uruguay \\
\hline $\begin{array}{l}\text { Comp_ } \\
\text { age }\end{array}$ & $1,9^{* * *}$ & $2,0^{* * *}$ & $1,9^{* * *}$ & $2,0^{* * *}$ & $1,8^{* * *}$ & $2,3^{* * *}$ & $2,0^{* * *}$ & $1,9^{* * *}$ \\
\hline Int_Age & $-1,1$ & $1,2^{* *}$ & $-1,5^{* * *}$ & $-1,0$ & $-1,4^{* * *}$ & $-1,1$ & $-1,3$ & $-1,4^{* * *}$ \\
\hline $\begin{array}{l}\text { Int_Hrs_ } \\
\text { In }\end{array}$ & $-2,3^{* * *}$ & $-2,7^{* * *}$ & $-2,0^{* * *}$ & $-1,9^{* * *}$ & $-1,5^{* * *}$ & $-3,3^{* * *}$ & $-3,3^{* * *}$ & $-3,5^{* * *}$ \\
\hline $\begin{array}{l}\text { Int_Hrs_ } \\
\text { Out }\end{array}$ & $1,4^{* * *}$ & $-1,1$ & $1,5^{* * *}$ & $1,5^{* * *}$ & $1,5^{* * *}$ & $1,7^{* * *}$ & $2,1^{* * *}$ & $1,8^{* * *}$ \\
\hline
\end{tabular}

*Significativo al $10 \%$; ${ }^{* *}$ significativo al $5 ;{ }^{* * *}$ significativo al $1 \%$.

Fuente: elaboración propia en base a OECD, 2015. 